\title{
Ising model with isotropic competing interactions in the presence of a field: A tricritical-Lifshitz-point realization
}

\author{
Marcia C. Barbosa \\ Instituto de Física, Universidade Federal do Rio Grande do Sul, Caixa Postal 15051, CEP 91501-970, \\ Porto Alegre, Rio Grande do Sul, Brazil
}

(Received 6 May 1993)

\begin{abstract}
We consider a spin system with competing interactions that are isotropic with respect to the axes of a cubic lattice. On the basis of an $\epsilon$ expansion, we demonstrate that for small values of the external field $H$, the paramagnetic-to-modulated-phase transition remains first order. For larger fields, such a transition changes to a continuous one at a tricritical point. As one varies the wave vector $q_{c}$ that is related to the modulated phase, one finds a line of such tricritical points. We remark that such a line must end at a Lifshitz tricritical point at $q_{c}=0$.
\end{abstract}

PACS number(s): 05.50.+q, 05.40.+j, 05.70.Fh

\section{INTRODUCTION}

The recent interest in the properties of modulated superstructures has stimulated the introduction of a wide variety of different models. For applications to magnetic compounds and other anisotropic systems, an Ising model with competing interactions along a single direction [axial next-nearest-neighbor Ising model (ANNNI)] was introduced and extensively studied [1]. Its phase diagram exhibits basically three phases (see Fig. 1): paramagnetic, ferromagnetic, and modulated phases. The modulated phase is characterized by an amplitude and a phase $q_{c}$. Both the paramagnetic-toferromagnetic and the paramagnetic-to-modulated-phase

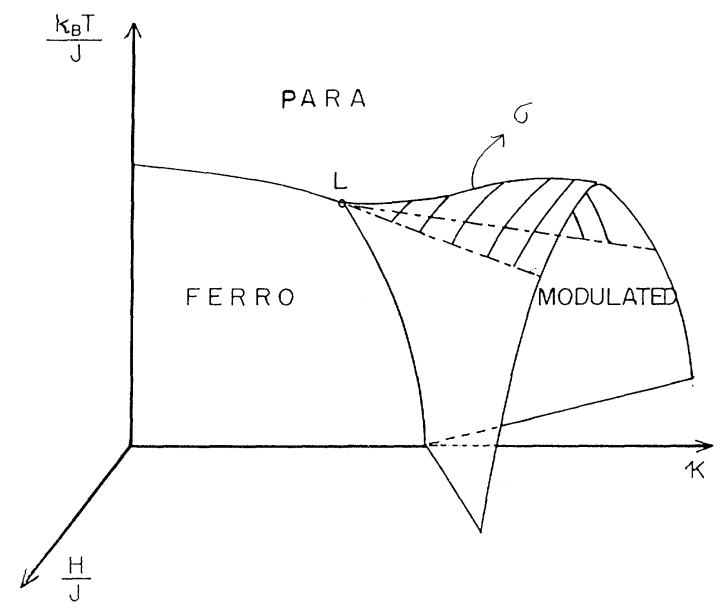

FIG. 1. Schematic phase diagram of the three-dimensional ANNNI model in the presence of an external field. Here a critical $\sigma$ surface is separated from a first-order transition by a line of tricritical points (dot-dashed) that ends at the Lifshitz point. Similar phase diagram is also obtained for the INNNI model when mean-field approximation is used. transitions are continuous (Ising-like and $X Y$-like, respectively). As one allows $q_{c} \rightarrow 0$, those two lines meet the ferromagnetic-to-modulated-phase first-order phase transition at a Lifshitz point.

Although this analysis provides an explanation of the presence of modulated phases, the model does not have the possibility of modulated order in more than one direction. Indeed, in the analogous structurally modulated phases observed in binary alloys $[2,3]$ we would normally expect the underlying couplings between the structural units to be isotropic. In order to repair this defect, Widom [4], as well as Upton and Yeomans [5,6], introduced an Ising model with isotropic competing interactions [an isotropic next-nearest-neighbor Ising (INNNImodel]. Within mean-field approximation and at the high-temperature region, they found a phase diagram quite similar to the phase diagram for the ANNNI but that it allows modulation in more than one fixed direction. This modulation arises in two forms: along any of the cubic axes (we will call it uniaxially modulated phase) or along any diagonal direction (cubically modulated phase). In order to check these results beyond mean-field approximation, we allowed fluctuations $[7,8]$. First, we noted that the possibility of different orientations for the modulated phase introduced new degrees of freedom. Consequently, in order to include them, following an usual procedure [9], we mapped the original model in a $2 m$-component spin model with cubic anisotropy within the $m$ components, where $2 m=6$ for the uniaxially-modulated phase while $2 m=8$ for the cubic modulated phase. The $2 m$-vectorial model exhibits a stable fixed point and that would usually lead to a well-defined critical behavior [10]. But, since the physical parameters of the original model were outside the domain of attraction of such a fixed point, no criticality would arise and the transition was found to be first order. Note that, in that case, fluctuations change drastically the mean-field results.

Unfortunately, this mapping cannot be applied close to the Lifshitz point where $q_{c} \rightarrow 0$ and, consequently, 
we were not able to guarantee that the transition would stay first order up to the "Lifshitz point" (of course, if the transition would stay first order all the way through the $q_{c}=0$ locus, the Lifshitz point would be an end point). We then decided to use another approach and, instead of using the mapping, we stayed in the vicinity of $q_{c} \approx 0$ and we employed a renormalization group in the $d=8-\epsilon$ expansion. In that case, we found a continuous transition that should indicate that the Lifshitz point should be critical $[7,11]$. This method is, as the previous approach, not satisfactory, since its results are valid close to $d=8$ and that it is far from the physical dimension $d=3$. In resume one cannot say, until now, what is the character of the Lifshitz point.

In this paper, we try to understand what happens close to that point. In order to do so, we analyze the effects on the paramagnetic-to-modulated phase transition when an external field $H$ is introduced.

In order to construct a phase diagram, we perform a mean-field analysis. Under that approximation, we show that the critical line, present in the absence of the external field, gives rise to a critical surface as one allows a nonzero external $H$. That surface meets a first-order phase boundary at a line of tricritical points. Such behavior is quite similar to the ANNNI case (see Fig. 1), which is not surprising since, even in zero external field, both phase diagrams exhibit some resemblance.

Next, we study what happens when one allows fluctuations. For low values of the external field $H<H_{t_{1}}$, we find that the first-order line, which we have obtained for $H=0[7,8]$, becomes a first-order surface symmetric in $H=0$. This phase boundary is limited by lines of tricritical points situated at critical fields $H= \pm H_{t_{1}}$. For $H>H_{t_{1}}$ a critical surface can be found. As one increases the external field even more one finds, at $|H|=H_{t_{2}}>H_{t_{1}}$, another line of tricritical points where the continuous surface finds another firstorder phase boundary. Both $H_{t_{1}} \rightarrow 0$ and $H_{t 2} \rightarrow 0$ as $q_{c} \rightarrow 0$, indicating that the Lifshitz point should be critical.

The layout of this paper is as follows. In Sec. II we introduce the model and we give its mean-field solution. In Sec. III, we discuss the Hamiltonian of this system in a form suitable for renormalization-group analysis. In particular, we employ a "folding process" that eliminates the irrelevant degrees of freedom. Different from the $H=0$ case, due to the presence of a nonzero external field, besides the $2 m$ (where $m=3$ or $m=4$ ) critical fields, we also keep other noncritical spin fields, associated with the uniform and second-harmonic components. Recursion relations to first order in $\epsilon=4-d$ expansion are explicitly obtained and a stable fixed point is found. Even though many noncritical fields are maintained due to the iterations of the renormalization-group transformations, many terms in the full Hamiltonian become irrelevant and a simple critical picture emerges. Indeed, in Sec. IV, we confirm this result since a partial trace over the renormalized Hamiltonian leads to a reduced very simple Hamiltonian. We show that this effective Hamiltonian is in the same universality class as the $2 m$-vectorial model which exhibits a stable fixed point and a well-known critical behavior. We then show that, due to the presence of the external field, the physical parameters, different from what happens in zero field, are inside the domain of attraction of such stable fixed point if $H_{t_{2}} \geq H \geq H_{t_{1}}$. In that case, a continuous transition arises; otherwise, if $H \leq H_{t_{1}}$ or $H \geq H_{t_{2}}$, it should be first order. We end Sec. IV with some conclusions.

\section{MODEL}

The Ising model with isotropic competing interactions can be described as follows. Each cubic lattice site $\mathbf{r}$ is occupied by an Ising spin $s(\mathbf{r})$ that interacts through nearest-neighbor interactions of strength $J$, next-nearestneighbor couplings along the cubic axes of strength $-\kappa_{1} J$, and next-nearest-neighbor interactions across the face diagonals $-\kappa_{2} J$. The Hamiltonian of such system is given by

$$
\mathcal{H}=-\frac{1}{2} \sum_{\mathbf{q}} s(\mathbf{q}) J(\mathbf{q}) s(-\mathbf{q})-H \sum_{\mathbf{q}} s(\mathbf{q})
$$

where $H$ is here an external field and the Fourier transform of $J\left(\mathbf{r}-\mathbf{r}_{\mathbf{1}}\right)$ is $J(\mathbf{q})$ given by

$$
\begin{aligned}
J(\mathbf{q}) & =2 J\left[\cos \left(q_{x}\right)+\cos \left(q_{y}\right)+\cos \left(q_{z}\right)\right] \\
& -2 \kappa_{1} J\left[\cos \left(2 q_{x}\right)+\cos \left(2 q_{y}\right)+\cos \left(2 q_{z}\right)\right] \\
& -4 \kappa_{2} J\left[\cos q_{x} \cos q_{y}+\cos q_{x} \cos q_{z}+\cos q_{y} \cos q_{z}\right] .
\end{aligned}
$$

In the absence of the external field $H$, this model was recently studied through mean-field approximation by Upton and Yeomans [6]. They found that, besides the usual paramagnetic and ferromagnetic phases, there is a modulated phase for $1-4 \kappa_{1}-4 \kappa_{2}<0$. This phase is characterized by long-range order and by a maximum value of $J(\mathbf{q})$ at $\mathbf{q}=\mathbf{q}_{c}$. Different from the ANNNI model, the wave vector in this model points along any axial $x, y$, or $z$ direction if $\kappa_{1}<\kappa_{2} / 2$ and along any diagonal direction if $\kappa_{1}>\kappa_{2} / 2$.

Let us now see what happens when one introduces an external field. In order to do so, we will employ a meanfield approximation. As usual with these types of models $[6,12]$, the free energy can be derived from the Bogoliubov inequality which leads to

$$
F=N^{-1} \sum_{\mathbf{r}}\left\{-k_{B} T \ln \left[2 \cosh \left(\sum_{\mathbf{r}_{1}} \beta J\left(\mathbf{r}-\mathbf{r}_{1}\right) m\left(\mathbf{r}_{1}\right)+\beta H\right)\right]+\frac{1}{2} \sum_{\mathbf{r}_{1}} m(\mathbf{r}) J\left(\mathbf{r}-\mathbf{r}_{1}\right) m\left(\mathbf{r}_{1}\right)\right\}
$$


where $m(\mathbf{r})=\langle s(\mathbf{r})\rangle_{0}$ is the magnetization $\left(\langle\rangle_{0}\right.$ is the ensemble average with respect to the trial Hamiltonian in the Bogoliubov inequality) specified by

$$
m(\mathbf{r})=\tanh \left(\beta \sum_{\mathbf{r}_{1}} J\left(\mathbf{r}-\mathbf{r}_{1}\right) m\left(\mathbf{r}_{1}\right)+\beta H\right) .
$$

At sufficiently high temperatures, this equation is fulfilled by a paramagnetic solution given by

$$
m_{0}=\tanh \left(\beta H+\beta m_{0} \sum_{\mathbf{r}_{1}} J\left(\mathbf{r}-\mathbf{r}_{1}\right)\right)
$$

and, consequently, it is convenient to write

$$
\tilde{m}(\mathbf{r})=\beta \sum_{\mathbf{r}_{\mathbf{1}}} J\left(\mathbf{r}-\mathbf{r}_{1}\right)\left[m\left(\mathbf{r}_{\mathbf{1}}\right)-m_{0}\left(\mathbf{r}_{\mathbf{1}}\right)\right]
$$

where a $\tilde{m} \neq 0$ characterizes the ordered phase. Now, for small values of the external field, the magnetization $m_{0}$ is also small. Also, close to criticality, the magnetization $\tilde{m}$ is also small and, consequently, one can linearize Eq. (3) to find

$$
\begin{aligned}
F= & F_{0}+\frac{1}{2} \sum_{\mathbf{q}} \tilde{m}(\mathbf{q}) \tilde{r}(\mathbf{q}) \tilde{m}(-\mathbf{q}) \\
& +w \sum_{\left\{\mathbf{q}_{i}\right\}} \tilde{m}\left(\mathbf{q}_{1}\right) \tilde{m}\left(\mathbf{q}_{2}\right) \tilde{m}\left(\mathbf{q}_{3}\right) \delta\left(\sum_{i} \mathbf{q}_{i}\right) \\
& +u \sum_{\left\{\mathbf{q}_{i}\right\}} \tilde{m}\left(\mathbf{q}_{1}\right) \tilde{m}\left(\mathbf{q}_{2}\right) \tilde{m}\left(\mathbf{q}_{3}\right) \tilde{m}\left(\mathbf{q}_{\mathbf{4}}\right) \delta\left(\sum_{i} \mathbf{q}_{i}\right) \\
& -\sum \tilde{h}(-\mathbf{q}) \tilde{m}(\mathbf{q}),
\end{aligned}
$$

where $F_{0}$ is independent of $m_{q}$ and the magnetization $m_{0}$ is given by the zero of $\tilde{h}$. The parameters in Eq. (7) are given by

$$
\begin{aligned}
\tilde{h} & =k_{B} T\left(H-\left(k_{B} T-J\right) m_{0}-\frac{m_{0}^{3}}{3}\right), \\
\tilde{r} & =k_{B} T\left(\frac{k_{B} T}{J(\mathbf{q})}-1+m_{0}^{2}\right) \\
w & =\frac{k_{B} T}{3} m_{0} \\
u & =\frac{k_{B} T}{12}
\end{aligned}
$$

From Eqs. (2) and (7), one sees that two phases can be found: a disordered phase $\tilde{m}=0$ at high temperatures $\left(T>T_{c}\right)$ and an ordered phase $\tilde{m} \neq 0$ at low temperatures $\left(T<T_{c}\right)$. Therefore a critical surface can be found at

$$
k_{B} T_{c}=\max _{\mathbf{q}}\left[J(\mathbf{q})\left(1-m_{0}^{2}\right)\right],
$$

where $q_{c}$ specifies the critical wave vector. If $1-4 \kappa_{1}-$ $4 \kappa_{2}>0, q_{c}=0$ and the ordered phase is ferromagnetic.
If, otherwise, $1-4 \kappa_{1}-4 \kappa_{2}<0$ and $\kappa_{2}>2 \kappa_{1}$, the vector $\mathbf{q}_{\mathbf{c}}$ is along the $x, y$, or $z$ direction and it is given by

$$
\cos q_{c}=\frac{1-4 \kappa_{2}}{4 \kappa_{1}}
$$

It is then clear that the critical modes $\tilde{m}\left(q_{c}\right)$ play the role of the Landau order parameter in this theory. Now, it is straightforward to minimize the free energy Eq. (7) with respect to the noncritical terms $\tilde{m}(q=0), \tilde{m}\left(2 q_{c}\right), \ldots$, which should be eliminated in favor of the critical variables $\tilde{m}\left(q_{c}\right)$. On doing this, one finds the free energy

$$
\begin{aligned}
F= & F_{0}+\sum_{\alpha} \tilde{m}\left(\mathbf{q}_{c}^{\alpha}\right) \tilde{r} \tilde{m}\left(-\mathbf{q}_{c}^{\alpha}\right) \\
& +\tilde{u} \sum_{\alpha}\left[\tilde{m}\left(\mathbf{q}_{c}^{\alpha}\right) \tilde{m}\left(-\mathbf{q}_{c}^{\alpha}\right)\right]^{2} \\
& +\tilde{v} \sum_{(\alpha \neq \beta)} \tilde{m}\left(\mathbf{q}_{c}^{\alpha}\right) \tilde{m}\left(-\mathbf{q}_{c}^{\alpha}\right) \tilde{m}\left(\mathbf{q}_{c}^{\beta}\right) \tilde{m}\left(-\mathbf{q}_{c}^{\beta}\right),
\end{aligned}
$$

where $\alpha, \beta=1,2,3$ indicate the $x, y$, or $z$ direction, respectively, and

$$
\begin{aligned}
& \tilde{u}=6 u-\frac{18 w^{2}}{\tilde{r}(0)}-\frac{9 w^{2}}{\tilde{r}\left(2 q_{c}, 0,0\right)}, \\
& \tilde{v}=12 u-\frac{18 w^{2}}{\tilde{r}(0)}-\frac{36 w^{2}}{\tilde{r}\left(q_{c}, q_{c}, 0\right)} .
\end{aligned}
$$

Minimization of this free energy leads to the following possible ordered phases. First, if $\tilde{u}<\tilde{v} / 2$, or else if

$$
\frac{2}{\tilde{r}\left(q_{c}, q_{c}, 0\right)}-\frac{1}{\tilde{r}(0,0,0)}-\frac{1}{\tilde{r}\left(2 q_{c}, 0,0\right)}<0
$$

the ordered phase will exhibit axial order since Eq. (9) leads to a nonzero value of $\tilde{m}\left( \pm q_{c}, 0,0\right) \neq 0$, $\tilde{m}\left(0, \pm q_{c}, 0\right) \neq 0$, or $\tilde{m}\left(0,0, \pm q_{c}\right) \neq 0$ (axial order). Note that here only one of the $\tilde{m}$ will be nonzero.

Now, if $\tilde{u}>\tilde{v} / 2$, or else if

$$
\frac{2}{\tilde{r}\left(q_{c}, q_{c}, 0\right)}-\frac{1}{\tilde{r}(0,0,0)}-\frac{1}{\tilde{r}\left(2 q_{c}, 0,0\right)}>0
$$

the ordered phase is characterized by nonzero values of $\tilde{m}\left( \pm q_{c}, 0,0\right) \neq 0, \tilde{m}\left(0, \pm q_{c}, 0\right) \neq 0$, and $\tilde{m}\left(0,0, \pm q_{c}\right) \neq 0$ (cubic order). In both cases, the critical surface meets the first-order phase boundary at a tricritical point at $\tilde{u}=0$. This line of tricritical points ends at the Lifshitz point that is a tricritical Lifshitz point. Note that this phase diagram is qualitatively similar to the phase diagram obtained in the ANNNI case (see Fig. 1) [12]. 


\section{THE RENORMALIZATION-GROUP APPROACH}

Now let us consider what happens when one allows fluctuations. On going to a continuous representation by adding a weighing term $w(s)$ for each spin, one finds a Hamiltonian given by

$$
\begin{aligned}
\overline{\mathcal{H}}= & -\frac{1}{2} \sum_{\mathbf{q}} s(\mathbf{q}) u_{2}(\mathbf{q}) s(-\mathbf{q}) \\
& -u_{4} \sum_{\mathbf{q}_{i}} s\left(\mathbf{q}_{1}\right) s\left(\mathbf{q}_{2}\right) s\left(\mathbf{q}_{3}\right) s\left(\mathbf{q}_{4}\right) \delta\left(\sum_{i} \mathbf{q}_{i}\right) \\
& +\sum_{\mathbf{q}} h(-\mathbf{q}) s(\mathbf{q})
\end{aligned}
$$

where, as usual, we have $\overline{\mathcal{H}}=-\mathcal{H} / k_{B} T-w(s)$ with $\mathcal{H}$ given by Eq. (1) and

$$
\begin{aligned}
u_{2} & =1-\frac{J(\mathbf{q})}{k_{B} T} \\
h & =k_{B} T H .
\end{aligned}
$$

Now, due to the presence of an external field, even in the paramagnetic phase a nonzero magnetization $m_{0}$ is present. As usual, in order to take fluctuations into account, we make an expansion about the paramagnetic phase. It seems thus natural to shift the spin variable $s(\mathbf{q})=m_{0}+\tilde{s}$. The Hamiltonian given by Eq. (15) then becomes

$$
\begin{aligned}
\overline{\mathcal{H}}= & \mathcal{H}_{0}+\tilde{\mathcal{H}} \\
\tilde{\mathcal{H}}= & -\frac{1}{2} \sum_{\mathbf{q}} \tilde{s}(\mathbf{q}) \tilde{r}(\mathbf{q}) \tilde{s}(-\mathbf{q}) \\
& -\tilde{w} \sum_{\left\{\mathbf{q}_{i}\right\}} \tilde{s}\left(\mathbf{q}_{1}\right) \tilde{s}\left(\mathbf{q}_{2}\right) \tilde{s}\left(\mathbf{q}_{3}\right) \delta\left(\sum_{i} \mathbf{q}_{i}\right) \\
& -\tilde{u_{4}} \sum_{\left\{\mathbf{q}_{i}\right\}} \tilde{s}\left(\mathbf{q}_{1}\right) \tilde{s}\left(\mathbf{q}_{2}\right) \tilde{s}\left(\mathbf{q}_{3}\right) \tilde{s}\left(\mathbf{q}_{4}\right) \delta\left(\sum_{i} \mathbf{q}_{i}\right) \\
& +\sum_{\mathbf{q}} \tilde{h}(-\mathbf{q}) \tilde{s}(\mathbf{q}),
\end{aligned}
$$

where the magnetization $m_{0}$ was chosen in order to have $\tilde{h}=0$, where

$$
\begin{aligned}
\tilde{r} & =u_{2}+12 u_{4} m_{0}^{2} \\
\tilde{w} & =4 u_{4} m_{0} \\
\tilde{h} & =h-u_{2} m_{0}-4 u_{4} m_{0}^{3}, \\
\tilde{u}_{4} & =u_{4}
\end{aligned}
$$

and where $\mathcal{H}_{0}$ does not depend on the spin variables.

Now that we have the Hamiltonian properly written, we need set out a renormalization-group program. Such program should be done by the "shell strategy." That process, usually applied for the paramagnetic-toferromagnetic-phase transitions, eliminates the irrelevant degrees of freedom related to the noncritical spins. Note that, in the present problem, for modulated phases, fluctuations with $\mathbf{q}$ close to $\mathbf{q}_{\mathbf{c}}$ will be critical and, in that case, will play an essential role. To take them into account, we introduce a new Brillouin zone given by $q_{i} \leq q_{c} / 2$ with $i=x, y, z$. Then, as usual $[9,7]$, we fold the original zone into the new one by (i) dividing the sums over $\mathbf{q}$ into pieces running from $\left(l_{i}-1\right) q_{c} / 2 \leq\left|q_{i}\right| \leq l_{i} q_{c} / 2$ with $i=x, y, z$ and with $l_{i}=1,2, \ldots$, and (ii) shifting the sums to run from $\left|q_{i}\right| \leq q_{c} / 2$ and defining new spin variables given by

$$
\begin{aligned}
\tilde{s}(0,0,0) & =\sigma_{0}, \\
\tilde{s}\left(q_{i} \pm q_{c}, 0,0\right) & =\frac{1}{(2)^{1 / 2}}\left(\sigma_{1}^{\alpha} \pm i \sigma_{2}^{\alpha}\right), \quad \alpha=1,2,3, i=x, y, z \\
\tilde{s}\left(q_{i} \pm q_{c}, q_{j} \pm q_{c}, 0\right) & =\frac{1}{(2)^{1 / 2}}\left(\sigma_{3}^{\alpha} \pm i \sigma_{4}^{\alpha}\right), \quad \alpha=1,2,3,(i, j)=(x, y),(x, z),(y, z) \\
\tilde{s}\left(q_{i} \pm q_{c}, q_{j} \mp q_{c}, 0\right) & =\frac{1}{(2)^{1 / 2}}\left(\sigma_{5}^{\alpha} \pm i \sigma_{6}^{\alpha}\right), \quad \alpha=1,2,3,(i, j)=(x, y),(x, z),(y, z) \\
\tilde{s}\left(q_{i} \pm 2 q_{c}, 0,0\right) & =\frac{1}{(2)^{1 / 2}}\left(\sigma_{7}^{\alpha} \pm i \sigma_{8}^{\alpha}\right), \quad \alpha=1,2,3, i=x, y, z
\end{aligned}
$$


where here, for simplicity, we are assuming modulation along $x, y$, or $z$ direction $\left(\kappa_{1}<\kappa_{2} / 2\right)$. Similar results are obtained when one studies the modulation along the diagonals $\left(\kappa_{1}>\kappa_{2} / 2\right)$.

After those steps, the Hamiltonian given by Eq. (15) maps into a new one given by

$$
\begin{aligned}
\tilde{\mathcal{H}}= & -\frac{1}{2} \sum_{\mathbf{q}}\left[\sigma_{0}(\mathbf{q}) \tilde{r_{0}}(\mathbf{q}) \sigma_{0}(-\mathbf{q})\right. \\
& \left.+\sum_{\mathbf{q} i, \alpha} \sigma_{i}^{\alpha}(\mathbf{q}) \tilde{r}\left(\mathbf{q}+\mathbf{q}_{i}^{\alpha}\right) \sigma_{i}^{\alpha}\left(\mathbf{q}_{c}\right)\right] \\
& -\tilde{w}_{0} \sum_{\left\{\mathbf{q}_{i}\right\}, \alpha} \sigma_{0}\left[\left(\sigma_{i}^{\alpha}\right)^{2}+\left(\sigma_{i+1}^{\alpha}\right)^{2}\right] \\
& -\tilde{w}_{1} \sum_{\left\{\mathbf{q}_{i}\right\}, \alpha, \beta}\left\{\sigma_{3}^{\alpha}\left[\sigma_{1}^{\alpha} \sigma_{1}^{\beta}+\sigma_{2}^{\alpha} \sigma_{2}^{\beta}\right]+\sigma_{4}^{\alpha}\left[\sigma_{2}^{\alpha} \sigma_{1}^{\beta}+\sigma_{1}^{\alpha} \sigma_{2}^{\beta}\right]\right. \\
& \left.+\sigma_{5}^{\beta}\left[\sigma_{1}^{\alpha} \sigma_{1}^{\beta}-\sigma_{2}^{\alpha} \sigma_{2}^{\beta}\right]+\sigma_{6}^{\beta}\left[\sigma_{2}^{\alpha} \sigma_{1}^{\beta}-\sigma_{1}^{\alpha} \sigma_{2}^{\beta}\right]\right\} \\
& -\tilde{w}_{2} \sum_{\left\{\mathbf{q}_{i}\right\}, \alpha}\left[\sigma_{7}^{\alpha}\left(\sigma_{1}^{\alpha}\right)^{2}-\sigma_{7}^{\alpha}\left(\sigma_{2}^{\alpha}\right)^{2}+2 \sigma_{8}^{\alpha} \sigma_{1}^{\alpha} \sigma_{2}^{\alpha}\right] \\
& -\tilde{u} \sum_{\left\{\mathbf{q}_{i}\right\}, \alpha}\left[\left(\sigma_{1}^{\alpha}\right)^{2}+\left(\sigma_{2}^{\alpha}\right)^{2}\right]^{2} \\
& \sum_{\left\{\mathbf{q}_{i}\right\}, \alpha, \beta}\left[\left(\sigma_{1}^{\alpha}\right)^{2}+\left(\sigma_{2}^{\alpha}\right)^{2}\right]\left[\left(\sigma_{1}^{\beta}\right)^{2}+\left(\sigma_{2}^{\beta}\right)^{2}\right]+O\left(\sigma^{4}\right) \\
& \\
& \\
&
\end{aligned}
$$

where $\alpha=1,2,3, \ldots$ specifies the wave vectors $\left(q_{c}, 0,0\right)$, $\left(q_{c}, q_{c}, 0\right),\left(2 q_{c}, 0,0\right), \ldots$ and

$$
\begin{aligned}
\tilde{r_{0}} & \equiv \tilde{r}(\mathbf{q}) \\
\tilde{r} & \equiv \tilde{r}\left(q_{i}+q_{c}, 0,0\right), \\
\tilde{r}_{1} & \equiv \tilde{r}\left(q_{i}+q_{c}, q_{j}+q_{c}, 0\right), \\
\tilde{r}_{2} & \equiv \tilde{r}\left(q_{i}+2 q_{c}, 0,0\right), \\
\tilde{w}_{0} & =\frac{\tilde{w}_{1}}{\sqrt{2}}=\sqrt{2} \tilde{w}_{2}=3 \tilde{w}, \\
\tilde{u} & =\frac{3}{2} u_{4}, \\
\tilde{v} & =3 u_{4} .
\end{aligned}
$$

Of course, we only considered contributions compatible with the wave-vector conservation and we omitted $i+j+$ $k>2$ terms since they turn out to be irrelevant under renormalization-group transformations.

Renormalization-group ( $\mathrm{RG}$ ) transformations are then generated in standard way $[13,12]$ leading to differential equations given by

$$
\begin{aligned}
\frac{d \tilde{u}}{d l}= & \epsilon \tilde{u}-40 \tilde{u}^{2}-8(m-1) \tilde{v}^{2}+24 \tilde{u} x_{0} \\
& +32 \tilde{u} x_{2}+8(m-1) \tilde{v} x_{1}-4 x_{0}^{2}-8 x_{2}^{2} \\
& -8 x_{0} x_{2}-2(m-1) x_{1}^{2} \\
\frac{d \tilde{v}}{d l}= & \epsilon \tilde{v}-32 \tilde{u} \tilde{v}-8 m \tilde{v}^{2}+40 \tilde{v} x_{0} \\
& +12 \tilde{v} x_{2}+16 \tilde{v} x_{1}+4 m \tilde{v} x_{1}-4 x_{0}^{2}-12 x_{0} x_{1} \\
& -8 x_{1} x_{2}-2\left(m-\frac{3}{2}\right) x_{1}^{2}, \\
\frac{d x_{0}}{d l}= & \epsilon x_{0}-32 \tilde{u} x_{0}-16(m-1) \tilde{v} x_{0}+16 x_{0} x_{2} \\
& +8(m-1) x_{0} x_{1}+8\left(1+\frac{m}{2}\right) x_{0}^{2} \\
\frac{d x_{1}}{d l} & =\epsilon x_{1}-16 \tilde{v} x_{1}+8 x_{0} x_{1}+6 x_{1}^{2} \\
\frac{d x_{2}}{d l}= & \epsilon x_{2}-16 \tilde{u} x_{2}+8 x_{0} x_{2}+4 x_{2}^{2},
\end{aligned}
$$

where $m=3$ for modulation along the $x, y$, or $z$ direction and $m=4$ for diagonal directions. On fixing $m=3$, one finds that the recursion relations exhibit a very simple form given by

$$
\begin{aligned}
\frac{d \bar{u}}{d l} & =\epsilon \bar{u}-40 \bar{u}^{2}-16 \bar{v}^{2}+24 \bar{u} x_{0}-8 x_{0} x_{2}, \\
\frac{d \bar{v}}{d l} & =\epsilon \bar{v}-32 \bar{u} \bar{v}-24 \bar{v}^{2}+24 \bar{v} x_{0}-4 x_{0}^{2}, \\
\frac{d x_{0}}{d l} & =\epsilon x_{0}-32 \bar{u} x_{0}-32 \bar{v} x_{0}+20 x_{0}^{2}, \\
\frac{d x_{1}}{d l} & =\epsilon x_{1}-16 \bar{v} x_{1}+8 x_{0} x_{1}-2 x_{1}^{2} \\
\frac{d x_{2}}{d l} & =\epsilon x_{2}-16 \tilde{u} x_{2}+8 x_{0} x_{2}-4 x_{2}^{2}
\end{aligned}
$$

where

$$
\begin{aligned}
& \bar{u}=\tilde{u}-\frac{x_{1}}{2}, \\
& \bar{v}=\tilde{v}-\frac{x_{2}}{2} .
\end{aligned}
$$

The RG equations as given by Eqs. (23) determine many fixed points. The critical behavior of the system is, nevertheless, specified by only the following:

$$
\begin{aligned}
\bar{u} & =\frac{\epsilon}{44}, \\
\bar{v} & =\frac{\epsilon}{88}, \\
x_{0} & =0, \quad x_{1}=x_{2}=0
\end{aligned}
$$

which we will call fixed point (a),

$$
\begin{aligned}
\bar{u} & =\frac{\epsilon}{56}, \\
\bar{v} & =\frac{\epsilon}{56}, \\
x_{0} & =0, \quad x_{1}=x_{2}=0,
\end{aligned}
$$

which we will call fixed point (b), 


$$
\begin{aligned}
\bar{u} & =\frac{\epsilon}{44}, \\
\bar{v} & =\frac{\epsilon}{88}, \\
x_{0} & =0, \quad x_{1}=\frac{9}{22} \epsilon, \quad x_{2}=\frac{7}{44} \epsilon,
\end{aligned}
$$

which we will call fixed point (c),

$$
\begin{aligned}
\bar{u} & =\frac{\epsilon}{56}, \\
\bar{v} & =\frac{\epsilon}{56}, \\
x_{0} & =0, \quad x_{1}=\frac{5}{14} \epsilon, \quad x_{2}=\frac{5}{28} \epsilon,
\end{aligned}
$$

which we will call fixed point $(d)$, and

$$
\bar{u}=\bar{v}=x_{0}=0, \quad x_{1}=\frac{\epsilon}{2}, x_{2}=\frac{\epsilon}{4},
$$

which we will call fixed point (e).

Linearization of the RG equations and the calculation of the corresponding eigenvalues and eigenvectors give the nature of those fixed points. The fixed point (a) exhibits three positive eigenvalues and consequently is stable in the $\left(u, v, x_{0}\right)$ subspace. The fixed point (b) is stable only in the subspace $\left(u=v, x_{0}\right)$. The fixed point (c) has five positive eigenvalues and, consequently, is stable; (d) has one negative eigenvalue related to the unstabilities present within the $(\bar{u}, \bar{v})$ subspace; (e) has three negative eigenvalues related to unstabilities in the $\left(\bar{u}, \bar{v}, x_{0}\right)$ plane.

What happens here? For $h=0$, as we already know $[7,8]$, even though there would be a stable fixed point [fixed point (a)], since the physical parameters, as can be seen from Eq. (21), are in the domain of the unstable fixed point (b), the transition is believed to be first order.

For $h \neq 0$, the fixed point (c) is the stable. In that case, if the physical parameters would be in the domain of (a) in the absence of external field, one would verify a crossover from (a) to (c) when the field would be applied. But this is not the case. One can see from Eqs. (21) and (25) that, for a small external field, namely $h<h_{t_{1}}$, the physical parameters are in the domain of the fixed points (b) and (d) (since $\bar{u}<\bar{v}$ ) and, consequently, for small fields a first order should be expected. As one increases the external field, the couplings $(\bar{u}, \bar{v})$ decrease and, precisely at $h=h_{t_{1}}$ specified by $\bar{u}=\bar{v}$, the system is able to reach the stable fixed point (c). At that tricritical point, the transition changes from first order to continuous. For $h>h_{t_{1}}$ there is a critical surface with a critical behavior specified by the exponents

$$
\begin{aligned}
& \nu=\frac{1}{2}+\frac{3}{22} \epsilon+\frac{605}{10548} \epsilon^{2}, \\
& \eta=\frac{5}{22} \epsilon^{2}
\end{aligned}
$$

Now, on increasing even more the external field, one finds at $h \geq h_{t_{2}}$, where $(\bar{u} \leq 0)$, a "critical runaway" associated with the flow to the unstable fixed point (e). At $h=h_{t_{2}}$, the tricritical point, associated with the Gaussian-like fixed point (e), leads to a first-order transition. Figure 2 illustrates this. Note that this last step

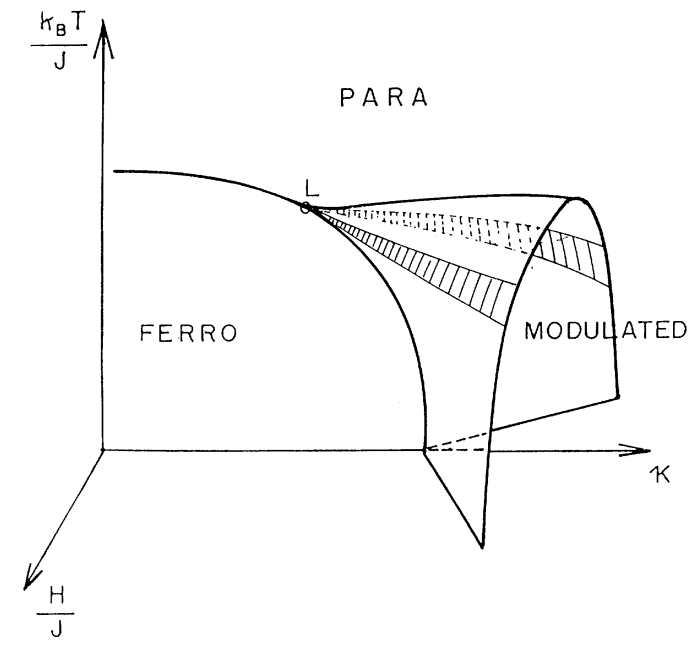

FIG. 2. Schematic phase diagram of the three-dimensional INNNI model in the presence of an external field. The first-order phase boundary present for small fields changes to continuous at a line $H=H_{t_{1}}$ of tricritical points. The continuous hatched surface is limited by two lines of tricritical fields, namely $H=H_{t_{1}}$ and $H=H_{t_{2}}$. Beyond the tricritical field $H>H_{t_{2}}$ the transition becomes first order.

is quite similar to what happens in the absence of fluctuations. In that sense one can argue that fluctuations are relevant only in the small field region.

\section{THE EFFECTIVE HAMILTONIAN}

Fluctuations can also be considered, using an alternative approach. One can argue that since the fields associated with wave vectors close to $(0,0,0),\left(2 q_{c}, 0,0\right)$, and $\left(q_{c}, q_{c}, 0\right)$ are noncritical, the spin fields related to them can be integrated out leading to an effective Hamiltonian given by

$$
\begin{aligned}
\tilde{\mathcal{H}}_{\mathrm{eff}} & =-\frac{1}{2} \sum_{\mathbf{q}} \sum_{i, \alpha}\left[\sigma_{i}^{\alpha}(\mathbf{q}) \tilde{r}_{\mathrm{eff}} \sigma_{i}^{\alpha}(\mathbf{q})\right] \\
& -\tilde{u}_{\mathrm{eff}} \sum_{\left\{\mathbf{q}_{i}\right\}, \alpha}\left[\left(\sigma_{1}^{\alpha}\right)^{2}+\left(\sigma_{2}^{\alpha}\right)^{2}\right]^{2} \\
& -\tilde{v}_{\mathrm{eff}} \sum_{\left\{\mathbf{q}_{i}\right\}, \alpha, \beta}\left[\left(\sigma_{1}^{\alpha}\right)^{2}+\left(\sigma_{2}^{\alpha}\right)^{2}\right]\left[\left(\sigma_{1}^{\beta}\right)^{2}+\left(\sigma_{2}^{\beta}\right)^{2}\right]
\end{aligned}
$$

with

$$
\begin{aligned}
& \tilde{r}_{\mathrm{eff}}=\tilde{r}+O\left(u_{4}, \tilde{w}^{2}\right) \\
& \tilde{u}_{\mathrm{eff}}=\frac{3 \tilde{u}_{4}}{2}-\frac{9 \tilde{w}^{2}}{4}\left[\frac{2}{\tilde{r}_{0}}-\frac{1}{\tilde{r}_{2}}\right] \\
& \tilde{v}_{\mathrm{eff}}=3 \tilde{u}_{4}-\frac{9 \tilde{w}^{4}}{2}\left[\frac{1}{\tilde{r}_{0}}-\frac{2}{\tilde{r}_{1}}\right]
\end{aligned}
$$

The above coefficients were obtained using diagram- 
matic techniques and treating the cubic terms perturbatively. Now one can see that this effective Hamiltonian is in the same universality class as the $2 m$-vectorial model with $m=3$ for $\kappa_{1}>\kappa_{2} / 2$ and $m=4$ otherwise. This model was studied by Mukamel and Krinsky [10,7] by means of renormalization-group transformation to second order in the $\epsilon=4-d$ expansion. They found the following fixed points:

$$
\begin{aligned}
& \text { (a) } \tilde{u}_{\text {eff }}^{\star}=\frac{\epsilon}{40}, \tilde{v}_{\text {eff }}^{\star}=0 \\
& \text { (b) } \tilde{u}_{\text {eff }}^{\star}=\frac{\epsilon}{4(m+8)}, \tilde{v}_{\text {eff }}^{\star}=\tilde{u}_{\text {eff }}^{\star}, \\
& \text { (c) } \tilde{u}_{\text {eff }}^{\star}=\frac{m-1}{8(5 m-4)} \epsilon, \tilde{v}_{\text {eff }}^{\star}=\frac{\epsilon}{8(5 m-4)},
\end{aligned}
$$

where only the last one is stable. Associated to that point, they computed critical exponents given by

$$
\begin{aligned}
\nu= & \frac{1}{2}+\frac{3}{4} \frac{(m-1)}{5 m-4} \epsilon \\
& +(m-1) \frac{\left(20 m^{2}+253 m-334\right)}{16(5 m-4)^{3}} \epsilon^{2}, \\
\eta & =\frac{(m-1)(2 m-1)}{4(5 m-4)^{2}} \epsilon^{2} .
\end{aligned}
$$

Let us consider again the $m=3$ ( axial order) case. Then, as we already stressed, the stable fixed point $u_{\text {eff }}^{\star}=2 v_{\text {eff }}^{\star}$ would be accessible only if the physical parameters $\tilde{u}$ and $\tilde{v}$ are inside its domain of attraction. In the absence of the external field, since $2 u_{\text {eff }}=v_{\text {eff }}$, this is never realized since the physical parameters are near the unstable fixed point (b). In that case, one usually assumes that no criticality could be found and the transition must be first order, confirming our results in Sec. III $[8,7]$.

On introducing an external field, one can see from Eq. (33) that, even though both couplings $\left(\tilde{u}_{\text {eff }}\right.$ and $\left.\tilde{v}_{\text {eff }}\right)$ decrease as $h$ increases, the ratio $\tilde{u}_{\text {eff }} / \tilde{v}_{\text {eff }}$ increases. In this sense, even if one begins with $\tilde{u}_{\text {eff }} / \tilde{v}_{\text {eff }}<1$ for $h=0$, one finds, for $h>h_{t_{1}}, \tilde{u}_{\text {eff }} / \tilde{v}_{\text {eff }} \geq 1$ and consequently the physical parameters are now near the stable fixed point (c). From Eq. (33), one sees that this last inequality implies that

$$
\tilde{u}_{4} \leq \frac{3}{2} \tilde{w}^{2}\left[\frac{4}{\tilde{r}_{1}}-\frac{1}{\tilde{r}_{2}}\right] .
$$

For these values of the physical parameters, the system exhibits a continuous transition with critical exponents given by Eq. (31). The tricritical field $h=h_{t_{1}} \equiv \beta H_{t_{1}}$, where the transition changes from first order to continuous, is related to the magnetization $m_{0}=m_{t_{1}}$ through Eq. (18) and it is completely specified by Eq. (36) when the equality holds.

On increasing even more the external field, for $h \geq h_{t_{2}}$, the physical parameters enter in the domain of attraction of the unstable fixed point (a), leading to a first-order transition. In that case, since $\tilde{v}_{\text {eff }}<0$, one has

$$
\tilde{u}_{4} \leq \frac{3}{2} w^{2}\left[\frac{1}{\tilde{r}_{0}}-\frac{2}{\tilde{r}_{1}}\right] .
$$

The tricritical point at $h=h_{t_{2}} \equiv \beta H_{t_{2}}$, the locus where the continuous transition associated to the fixed point $(a)$ becomes first order, is associated with the magnetization $m_{0}=m_{t_{2}}$ by Eq. (18) and is specified by Eq. (37) when equality holds.

Both tricritical loci depend on the wave vector $q_{c}$ through the values of $\left(r_{0}, r_{1}, r_{2}\right)$. As one varies $q_{c}$, both tricritical points originate two lines of tricritical points. One can now ask what does happen when on decreasing $q_{c}$, one approaches the Lifshitz point. On computing both Eqs. (36) and (37), one finds that both fields decrease smoothly $\left(h_{t_{1}} \rightarrow 0\right.$ and $\left.h_{t_{2}} \rightarrow 0\right)$ as the critical field approaches to zero $\left(q_{c} \rightarrow 0\right)$.

In resume, we have studied the Ising model with isotropic competing interactions in the presence of an external field. We first analyzed the model through meanfield approximation and we show that, in that case, the paramagnetic-to-modulated-continuous-phase transition present in the absence of a field becomes a critical surface limited by a tricritical line. This line ends at the Lifshitz point. This behavior is quite similar to the ANNNI case, leading us to the belief that the mean-field approach does not differentiate isotropic from anisotropic cases.

Next, we discussed what happens when one allows fluctuations. By renormalization-group analysis, we confirmed our previous result that at zero field the transition is fluctuation-induced first order. In the presence of a small field, this first-order line becomes a surface. At the tricritical field $h=h_{t_{1}}$ the transition changes to continuous. On increasing the even more external field, one finds at $h=h_{t_{2}}$ another tricritical locus where the transition changes again to first order. On varying the critical wave vector, both $h=h_{t_{1}}$ and $h=h_{t_{2}}$ originate lines of tricritical points. Those lines end at the Lifshitz point $\left(q_{c}=0\right)$ which, in that case, should be a tricritical Lifshitz point. Although our calculations were done only for modulations along the $x, y$, or $z$ direction $\left(\kappa_{1}<\kappa_{2} / 2\right)$, the same results are obtained for modulation along the diagonals $\left(\kappa_{1}>\kappa_{2} / 2\right)$.

Unfortunately, since the folding process is not valid for $\kappa_{1}=\kappa_{2} / 2$, we cannot apply them to the microemulsion limit. In that case, another diagrammatic procedure must be applied [14-16].

\section{ACKNOWLEDGMENTS}

I thank Professor M. D. Coutinho-Filho, Professor S. R. Salinas, and Professor C. S. O. Yokoi for introducing me to the method of solving the problem. Special thanks are due to Claudio M. Rizzato for useful discussions. This work was supported in part by Conselho Nacional de Desenvolvimento Científico e Tecnológico and Financiadora de Estudos e Projetos, Brazil. 
[1] W. Selke, in Phase Transition and Critical Phenomena, edited by C. Domb and J. L. Lebowitz (Academic, New York, 1992), Vol. 15, p.1.

[2] A. Loiseau, G. van Tendeloo, R. Portier, and F. Ducastelle, J. Phys. (Paris) 46, 595 (1985).

[3] D. Broddin, G. van Tendeloo, J. van Landuyt, S. Amelinckx, R. Porther, M. Guymont, and A. Loisaeu, Philos. Mag. A 54, 395 (1986).

[4] B. Widom, J. Chem. Phys. 84, 6943 (1986).

[5] J. M. Yeomans, in Solid State Physics, edited by H. Ehrenreich, F. Seitz, and D. Turnbull (Academic, New York, 1988), Vol. 41, p.151.

[6] P. J. Upton and J. M. Yeomans, Europhys. Lett. 5, 575 (1988); Phys. Rev. B 40, 479 (1989).

[7] Marcia C. Barbosa, Phys. Rev. B 42, 6363 (1990).

[8] Y. Levin and K. A. Dawson, Phys. Rev. A 42, 1976 (1990).

[9] M. Droz and M. D. Coutinho-Filho, in Magnetism and
Magnetic Materials, Philadelphia, 1975, Proceedings of the 21st Annual Conference on Magnetism and Magnetic Materials, AIP Conf. Proc. No. 29, edited by J. J. Becker, G. H. Lander, and J. J. Rhyne ( AIP, New York, 1976), p. 465.

[10] D. Mukamel and S. Krinsky, Phys. Rev. B 13, 5078 (1976).

[11] D. Mukamel and G. Grinstein, Phys. Rev. B 25, 381 (1982).

[12] C. S. O. Yokoi, M. D. Coutinho-Filho, and S. R. Salinas, Phys. Rev. B 31, 4502 (1985).

[13] David R. Nelson and Michael E. Fisher, Phys. Rev. B 11, 1030 (1975).

[14] Marcia C. Barbosa, Phys. Rev. B 46, 3672 (1992).

[15] Y. Levin, C. J. Mundy, and K. A. Dawson, Phys. Rev. A 45, 7309 (1992).

[16] Marcia C. Barbosa (unpublished). 